\title{
Superconductivity Provides Access to the Chiral Magnetic Effect of an Unpaired Weyl Cone
}

\author{
T. E. O’Brien, ${ }^{1}$ C. W. J. Beenakker, ${ }^{1}$ and İ. Adagideli ${ }^{2,1, *}$ \\ ${ }^{1}$ Instituut-Lorentz, Universiteit Leiden, P.O. Box 9506, 2300 RA Leiden, The Netherlands \\ ${ }^{2}$ Faculty of Engineering and Natural Sciences, Sabanci University, Orhanli-Tuzla, Istanbul 34956, Turkey
}

(Received 6 February 2017; published 19 May 2017)

\begin{abstract}
The massless fermions of a Weyl semimetal come in two species of opposite chirality, in two cones of the band structure. As a consequence, the current $j$ induced in one Weyl cone by a magnetic field $B$ [the chiral magnetic effect (CME)] is canceled in equilibrium by an opposite current in the other cone. Here, we show that superconductivity offers a way to avoid this cancellation, by means of a flux bias that gaps out a Weyl cone jointly with its particle-hole conjugate. The remaining gapless Weyl cone and its particle-hole conjugate represent a single fermionic species, with renormalized charge $e^{*}$ and a single chirality \pm set by the sign of the flux bias. As a consequence, the CME is no longer canceled in equilibrium but appears as a supercurrent response $\partial j / \partial B= \pm\left(e^{*} e / h^{2}\right) \mu$ along the magnetic field at chemical potential $\mu$.
\end{abstract}

DOI: 10.1103/PhysRevLett.118.207701

Introduction.-Massless spin-1/2 particles, the so-called Weyl fermions, remain unobserved as elementary particles, but they have now been realized as quasiparticles in a variety of crystals known as Weyl semimetals [1-5]. Weyl fermions appear in pairs of left-handed and right-handed chirality, occupying a pair of cones in the Brillouin zone. The pairing is enforced by the chiral anomaly [6]: A magnetic field induces a current of electrons in a Weyl cone, flowing along the field lines in the chiral zeroth Landau level. The current in the Weyl cone of one chirality has to be canceled by a current in the Weyl cone of opposite chirality to ensure zero net current in equilibrium. The generation of an electrical current density $\boldsymbol{j}$ along an applied magnetic field $\boldsymbol{B}$, the so-called chiral magnetic effect (CME) [7,8], has been observed as a dynamic, nonequilibrium phenomenon [9-13] - but it cannot be realized in equilibrium because of the fermion doubling [14-24].

Here, we present a method by which single-cone physics may be accessed in a superconducting Weyl semimetal, allowing for observation of the CME in equilibrium. The geometry is shown in Fig. 1. Application of a flux bias gaps out all but a single particle-hole conjugate pair of Weyl cones, of a single chirality \pm set by the sign of the flux bias. At nonzero chemical potential $\mu$, one of the two Weyl points sinks in the Cooper pair sea, the chiral anomaly is no longer canceled, and we find an equilibrium response $\partial j / \partial B= \pm\left(e^{*} e / h^{2}\right) \mu$, with $e^{*}$ the charge expectation value at the Weyl point.

We stress that the CME in a superconductor is not in violation of thermodynamics, which only demands a vanishing heat current in equilibrium. Indeed, in previous work on magnetically induced currents [25-27], it was shown that the fundamental principles of Onsager symmetry and gauge invariance forbid a linear relation between $\boldsymbol{j}$ and $\boldsymbol{B}$ in equilibrium. However, in a superconductor, the gauge symmetry is broken at a fixed phase of the order parameter, opening the door for the CME.

Pathway to single-cone physics.-First, we explain the mechanism by which a superconductor provides access to single-cone physics. A pair of Weyl cones at momenta $\pm \boldsymbol{k}_{0}$ of opposite chirality has the Hamiltonian [28]

$\mathcal{H}=\frac{1}{2} v_{F} \sum_{\boldsymbol{k}}\left[\psi_{\boldsymbol{k}}^{\dagger}\left(\boldsymbol{k}-\boldsymbol{k}_{0}\right) \cdot \boldsymbol{\sigma} \psi_{\boldsymbol{k}}-\phi_{\boldsymbol{k}}^{\dagger}\left(\boldsymbol{k}+\boldsymbol{k}_{0}\right) \cdot \boldsymbol{\sigma} \phi_{\boldsymbol{k}}\right]$,

where $\boldsymbol{k} \cdot \boldsymbol{\sigma}=k_{x} \sigma_{x}+k_{y} \sigma_{y}+k_{z} \sigma_{z}$ is the sum over Pauli matrices acting on the spinor operators $\psi$ and $\phi$ of lefthanded and right-handed Weyl fermions. The Fermi velocity is $v_{F}$ and we set $\hbar \equiv 1$ (but keep $h$ in the formula for the CME).

If $\mathcal{H}$ would be the Bogoliubov-de Gennes (BDG) Hamiltonian of a superconductor, particle-hole symmetry

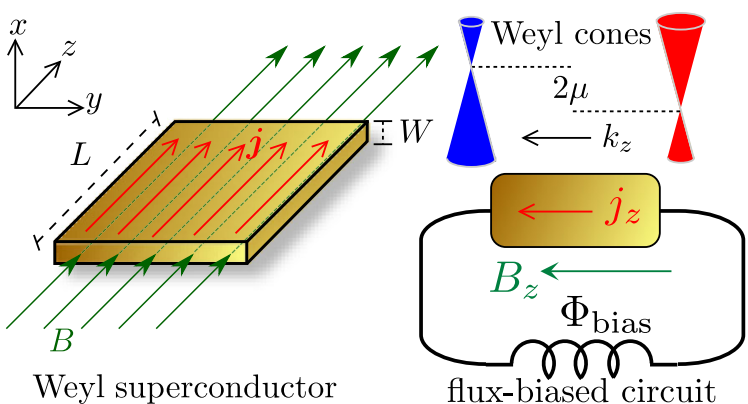

FIG. 1. Left panel: Slab of a Weyl superconductor subject to a magnetic field $B$ in the plane of the slab (thickness $W$ less than the London penetration depth). The equilibrium chiral magnetic effect manifests itself as a current response $\partial j / \partial B= \pm \kappa(e / h)^{2} \mu$ along the field lines, with $\kappa$ a charge renormalization factor and $\mu$ the equilibrium chemical potential. The right panel shows the fluxbiased measurement circuit and the charge-conjugate pair of Weyl cones responsible for the effect, of a single chirality \pm determined by the sign of the flux bias. 
would require that $\phi_{k}=\sigma_{y} \psi_{-k}^{\dagger}$. With the help of the matrix identity $\sigma_{y} \sigma_{\alpha} \sigma_{y}=-\sigma_{\alpha}^{*}$ and the anticommutator $\psi \sigma_{\alpha}^{*} \psi^{\dagger}=$ $-\psi^{\dagger} \sigma_{\alpha} \psi$, we rewrite Eq. (1) as

$$
\begin{aligned}
\mathcal{H} & =\frac{1}{2} v_{F} \sum_{\boldsymbol{k}}\left[\psi_{\boldsymbol{k}}^{\dagger}\left(\boldsymbol{k}-\boldsymbol{k}_{0}\right) \cdot \boldsymbol{\sigma} \psi_{\boldsymbol{k}}-\psi_{-\boldsymbol{k}}^{\dagger}\left(\boldsymbol{k}+\boldsymbol{k}_{0}\right) \cdot \boldsymbol{\sigma} \psi_{-\boldsymbol{k}}\right] \\
& =v_{F} \sum_{\boldsymbol{k}} \psi_{\boldsymbol{k}}^{\dagger}\left(\boldsymbol{k}-\boldsymbol{k}_{0}\right) \cdot \boldsymbol{\sigma} \psi_{\boldsymbol{k}},
\end{aligned}
$$

producing a single-cone Hamiltonian. Then, if we hypothetically impose a magnetic field $\boldsymbol{B}=\nabla \times \boldsymbol{A}$ via $\boldsymbol{k} \mapsto \boldsymbol{k}-\boldsymbol{e A}$, the zeroth Landau level carries a current density $\boldsymbol{j}=(e / h)^{2} \mu \boldsymbol{B}$ in an energy interval $\mu$. This is the chiral anomaly of an unpaired Weyl cone [6].

Model Hamiltonian of a Weyl superconductor.-As a minimal model for single-cone physics, we consider the BDG Hamiltonian [29]

$$
\begin{aligned}
& \mathcal{H}=\sum_{k} \Psi_{k}^{\dagger} H(\boldsymbol{k}) \Psi_{k}, \quad \Psi_{k}=\left(\psi_{k}, \sigma_{y} \psi_{-k}^{\dagger}\right), \\
& H(\boldsymbol{k})=\left(\begin{array}{cc}
H_{0}(\boldsymbol{k}-e \boldsymbol{A}) & \Delta_{0} \\
\Delta_{0}^{*} & -\sigma_{y} H_{0}^{*}(-\boldsymbol{k}-e \boldsymbol{A}) \sigma_{y}
\end{array}\right), \\
& H_{0}(\boldsymbol{k})=\sum_{\alpha} \tau_{z} \sigma_{\alpha} \sin k_{\alpha}+\tau_{0}\left(\beta \sigma_{z}-\mu \sigma_{0}\right)+m_{\boldsymbol{k}} \tau_{x} \sigma_{0}, \\
& m_{k}=m_{0}+\sum_{\alpha}\left(1-\cos k_{\alpha}\right) \text {. }
\end{aligned}
$$

This is a tight-binding model on a simple cubic lattice (lattice constant $a_{0} \equiv 1$, nearest-neighbor hopping energy $t_{0} \equiv 1$, electron charge $+e$ ). The Pauli matrices $\tau_{\alpha}$ and $\sigma_{\alpha}$, with $\alpha \in\{x, y, z\}$, act, respectively, on the orbital and spin degree of freedom. (The corresponding unit matrices are $\tau_{0}$ and $\sigma_{0}$.) Time-reversal symmetry is broken by a magnetization $\beta$ in the $z$ direction, $\mu$ is the chemical potential, $\boldsymbol{A}$ the vector potential, and $\Delta_{0}$ is the $s$-wave pair potential.

The single-electron Hamiltonian $H_{0}$ in the upper-left block of $H$ is the four-band model [14,30] of a Weyl semimetal formed from a topological insulator in the $\mathrm{Bi}_{2} \mathrm{Se}_{3}$ family, layered in the $x-y$ plane. For a small mass term $m_{0}<\beta$, it has a pair of Weyl cones centered at $\left(0,0, \pm \sqrt{\beta^{2}-m_{0}^{2}}\right)$, displaced in the $k_{z}$ direction by the magnetization. (We retain inversion symmetry, so the Weyl points line up at the same energy.) A coupling of this pair of electron Weyl cones to the pair of particle-hole conjugate Weyl cones in the lower-right block of $H$ is introduced by the pair potential, which may be realized by alternating the layers of the topological insulator with a conventional BCS superconductor [31,32]. (Intrinsic superconducting order in a doped Weyl semimetal, with more unconventional pair potentials, is an alternative possibility [33-42].) The superconductor does not gap out the Weyl cones if $\Delta_{0}<\sqrt{\beta^{2}-m_{0}^{2}}$.
Flux bias into the single-cone regime.-As explained by Meng and Balents [31], a Weyl superconductor has topologically distinct phases characterized by the number $\mathcal{N} \in\{2,1,0\}$ of ungapped particle-hole conjugate pairs of Weyl cones. We propose to tune through the phase transitions in an externally controllable way by means of a flux bias, as shown in the circuit of Fig. 1. For a real $\Delta_{0}>0$, the flux bias $\Phi_{\text {bias }}$ enters in the Hamiltonian via the vector potential component $A_{z}=\Phi_{\text {bias }} / L \equiv \Lambda / e$. The $\Phi_{\text {bias }}$-dependent band structure is shown in Fig. 2, calculated [43] in a slab geometry with hard-wall boundaries at $x= \pm W / 2$ and periodic boundary conditions at $y= \pm W^{\prime} / 2$ (sending $W^{\prime} \rightarrow \infty$ ).

The two pairs of particle-hole conjugate Weyl cones are centered at $\left(0,0, K_{ \pm}\right)$and $\left(0,0,-K_{ \pm}\right)$, with

$$
K_{ \pm}^{2}=\left(\sqrt{\beta^{2}-m_{0}^{2}} \pm \Lambda\right)^{2}-\Delta_{0}^{2} .
$$

We have assumed $\Lambda, K_{ \pm} \ll 1$, so the Weyl cones are near the center of the Brillouin zone. A cone is gapped when $K_{ \pm}$ becomes imaginary; hence, the $\mathcal{N}=1$ phase is entered with increasing $\Lambda>0$ when

$$
\sqrt{\beta^{2}-m_{0}^{2}}+\Lambda>\Delta_{0}>\left|\sqrt{\beta^{2}-m_{0}^{2}}-\Lambda\right| .
$$

This is the regime in which we can observe the CME of an unpaired Weyl cone, as we will show in the following.

Magnetic response of a unpaired Weyl cone.-We assume that the slab is thinner than the London penetration depth so that we can impose an unscreened magnetic field $B_{z}$ in the $z$ direction $[44,45]$. The vector potential, including the flux bias, is $\boldsymbol{A}=\left(0, x B_{z}, \Lambda / e\right)$. To explain in the

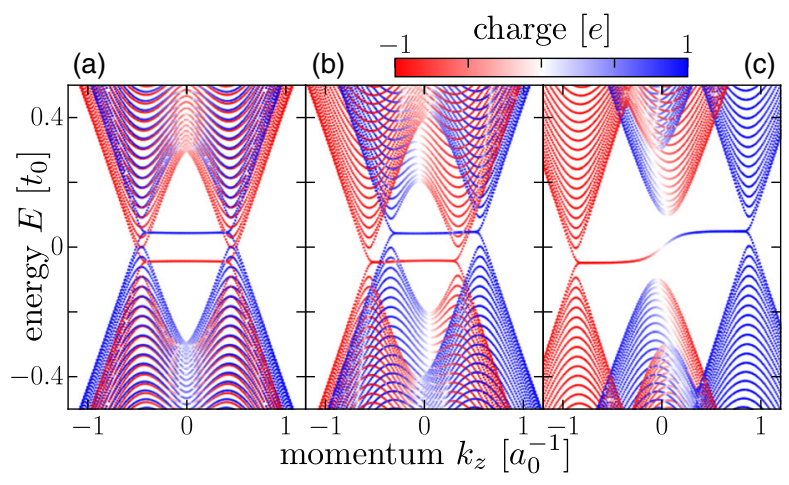

FIG. 2. Effect of a flux bias on the band structure of a Weyl superconductor. The plots are calculated from the Hamiltonian (3) in the slab geometry of Fig. 1 (parameters: $m_{0}=0, \Delta_{0}=0.2$, $\left.\beta=0.5, \mu=-0.05, k_{y}=0, W=100, B_{z}=0\right)$. The color scale indicates the charge expectation value, to distinguish electronlike and holelike cones. As the flux bias is increased from $\Lambda=0$ in panel (a), to $\Lambda=0.1$ and 0.4 in panels (b) and (c), one electronhole pair of Weyl cones merges and is gapped by the pair potential. What remains in panel (c) is a single pair of chargeconjugate Weyl cones, connected by a surface Fermi arc. This is the phase that supports a chiral magnetic effect in equilibrium. 
simplest terms how single-cone physics emerges, we linearize in $\boldsymbol{k}$ and $\boldsymbol{A}$ and set $m_{0}=0$, so the mass term $m_{k}$ can be ignored. (All nonlinearities will be fully included later on [46]).

The Hamiltonian (3) is approximately block diagonalized by the Bogoliubov transformation

$$
\begin{aligned}
\tilde{\psi}_{k} & =\cos \left(\theta_{k} / 2\right) \psi_{k}+i \sin \left(\theta_{k} / 2\right) \tau_{z} \sigma_{x} \psi_{-k}^{\dagger}, \\
\tilde{H} & =U^{\dagger} H U, \quad U=\exp \left(\frac{1}{2} i \theta_{k} \nu_{y} \tau_{z} \sigma_{z}\right),
\end{aligned}
$$

where the Pauli matrix $\nu_{\alpha}$ acts on the particle-hole degree of freedom. If we choose the $k_{z}$-dependent angle $\theta_{k}$ such that

$$
\begin{aligned}
\cos \theta_{k} & =-\sin k_{z} / \Delta_{k}, \quad \sin \theta_{k}=\Delta_{0} / \Delta_{k}, \\
\Delta_{k} & =\sqrt{\Delta_{0}^{2}+\sin ^{2} k_{z}},
\end{aligned}
$$

the gapless particle-hole conjugate Weyl points at $k_{z}^{2}=$ $K_{+}^{2} \approx 2 \Delta_{0}\left(\beta+\Lambda-\Delta_{0}\right) \ll 1$ are predominantly contained in the $(\nu, \tau)=(-,-)$ block of $\tilde{H}$. Projection onto this block gives the low-energy Hamiltonian

$$
\tilde{\mathcal{H}}=\sum_{\boldsymbol{k}} \tilde{\psi}_{\boldsymbol{k}}^{\dagger}\left[\sum_{\alpha} v_{\alpha}\left(\delta k_{\alpha}-q_{\alpha} A_{\alpha}\right) \sigma_{\alpha}-q_{0} \mu \sigma_{0}\right] \tilde{\psi}_{\boldsymbol{k}},
$$

where $\quad \boldsymbol{k}=\left(0,0, K_{+}\right)+\delta \boldsymbol{k}, \quad \boldsymbol{v}=(1,1,-\kappa), \quad q_{0}=\kappa$, $\boldsymbol{q}=(\kappa e, \kappa e, e / \kappa)$, and

$$
\kappa \approx K_{+} / \sqrt{\Delta_{0}^{2}+K_{+}^{2}}=\sqrt{1-\Delta_{0}^{2} /(\beta+\Lambda)^{2}} .
$$

Equation (8) represents a single-cone Hamiltonian of the form (2), with a renormalized velocity $v_{\alpha}$ and charge $q_{\alpha}$. As a consequence, the CME formula for the equilibrium current density $j_{z}$ is renormalized into [47]

$$
\frac{\partial j_{z}}{\partial B_{z}}=\frac{q_{y} q_{z}}{h^{2}} q_{0} \mu=\frac{e^{*} e}{h^{2}} \mu, \quad e^{*}=\kappa e .
$$

The renormalization of $v$ does not enter because the CME is independent of the Fermi velocity. One can understand why the product $e^{*} e$ appears rather than the more intuitive $\left(e^{*}\right)^{2}$, by noticing that $\partial j_{z} / \partial B_{z}$ changes sign upon inversion of the momentum-hence, only odd powers of $\kappa \propto K_{+}$are permitted.

Consistency of a nonzero equilibrium electrical current and vanishing particle current.-For thermodynamic consistency, to avoid heat transport at zero temperature, the CME should not produce a particle current in the superconductor. The flow of charge $e^{*}$ particles in the $z$ direction should, therefore, be canceled by a charge-neutral counterflow. This counterflow is provided by the surface Fermi arc, as illustrated in Fig. 3. The Fermi arc is the band of surface states connecting the Weyl cones $[48,50]$, to ensure that the chirality of the zeroth Landau level does not produce an excess number of left movers over right movers. In a Weyl superconductor, one can distinguish a trivial or nontrivial connectivity, depending on whether the Fermi arc connects cones of the same or of opposite charge [29,51]. Here, the connectivity is necessarily nontrivial because there is only a single pair of charge-conjugate Weyl cones. As a consequence, the Fermi arc is approximately charge neutral near the Fermi level (near $E=0$ ), so it can cancel the particle current without canceling the charge current [52].

We stress the essential role played by superconductivity, which separates the electronic transport of heat from the transport of charge: A cancellation of a particle current in the bulk by a particle current at the surface is possible without superconductivity, but then, the charge current is also canceled. (For such a spatial separation of counterpropagating particle currents in the normal state, see Refs. [53-55]).

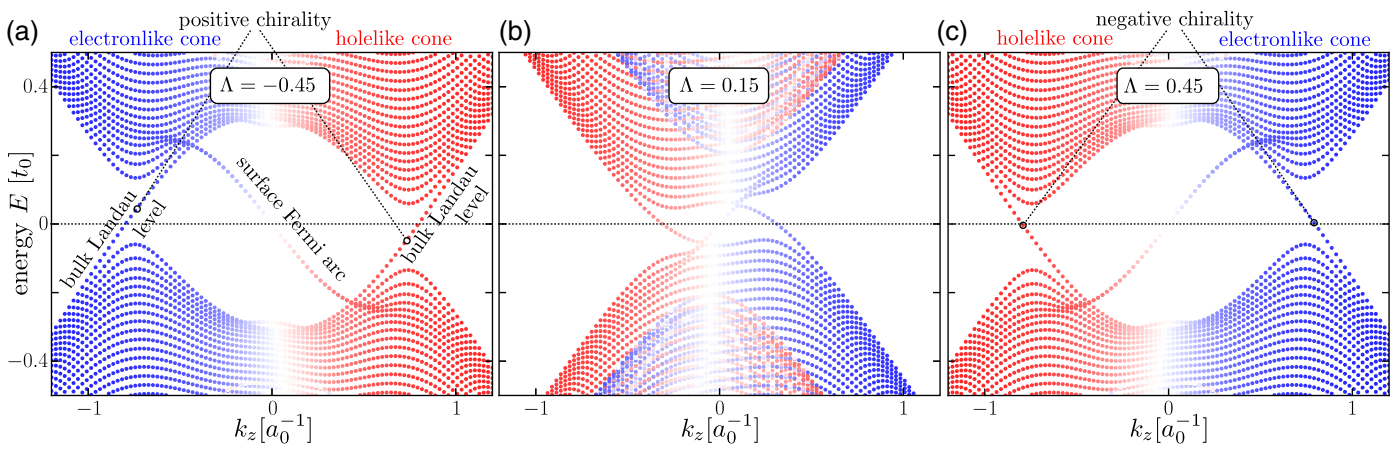

FIG. 3. Chirality switch of a pair of charge-conjugate Weyl cones, induced by a sign change of the flux bias $\Lambda=-0.45,0.15$, and 0.45 in panels (a), (b), and (c), respectively. All other parameters are the same in each panel: $m_{0}=0, \Delta_{0}=0.6, \beta=0.5, W=100, k_{y}=0$, $\mu=-0.05$, and $B_{z}=0.001 a_{0}^{-2} h / e$. The charge color scale of the band structure is as in Fig. 2. Particles in the zeroth Landau level propagate through the bulk in the same direction both in the electronlike cone and in the holelike cone, as determined by the chirality $\chi=-\operatorname{sign} \Lambda$ [49]. A net charge current appears in equilibrium because $\mu<0$, so there is an excess of electronlike states at $E>0$. [States at $E<0$ do not contribute to the equilibrium current (11).] The particle current is canceled by the Fermi arc that connects the chargeconjugate Weyl cones. The Fermi arc carries an approximately neutral current; hence, the charge current in the chiral Landau level is not much affected by the counterflow of particles in the Fermi arc. 


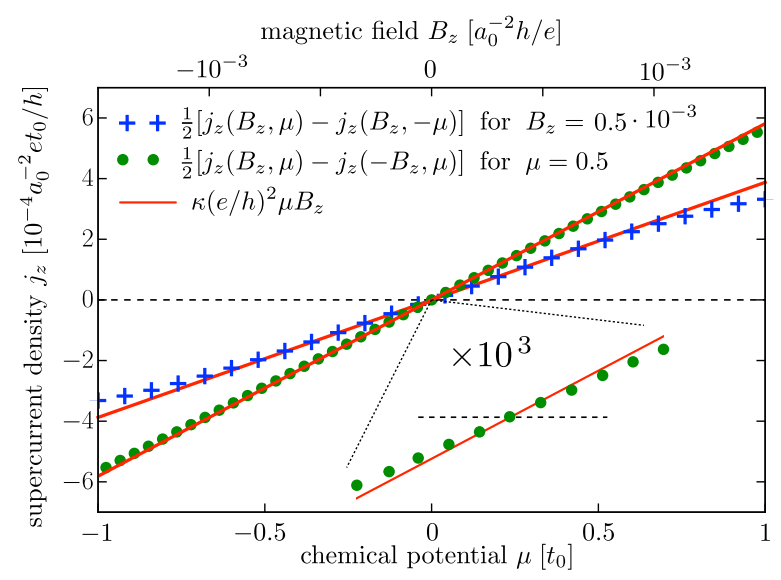

FIG. 4. Data points: numerical calculation of the equilibrium supercurrent in the flux-biased circuit of Fig. 1. The parameters are $m_{0}=0, \Delta_{0}=0.6, \quad \beta=0.5, \quad \Lambda=0.45, \quad W=100$, and $k_{\mathrm{B}} T=0.01$; the green data points are for a fixed $\mu$ with a variation of $B_{z}$ and the blue points are for a fixed $B_{z}$ with a variation of $\mu$. The data are antisymmetrized, as indicated, to eliminate the background supercurrent from the flux bias. The solid curves are the analytical prediction (10), with $\kappa=0.775$ following directly from Eq. (9) (no fit parameters). The $B_{z^{-}}$ dependent data is also shown with a zoom in to very small magnetic fields, down to $10^{-7} a_{0}^{-2} h / e$, to demonstrate that the linear $B_{z}$ dependence continues when $l_{m}>W$.

Numerical simulation.-We have tested these analytical considerations in a numerical simulation of the model Hamiltonian (3), in the slab geometry of Fig. 1. At temperature $T$, the equilibrium current is given by [56]

$$
I_{z}=\frac{1}{2} \sum_{n, m} \int \frac{d k_{z}}{2 \pi} \tanh \left(\frac{E_{n m}}{2 k_{\mathrm{B}} T}\right) \Theta\left(E_{n m}\right) \frac{\partial E_{n m}}{\partial A_{z}},
$$

where $\Theta(E)$ is the unit step function and the prefactor of $1 / 2$ takes care of a double counting in the BDG Hamiltonian $H$. The eigenvalues $E_{n m}\left(k_{z}\right)$ of $H$ are labeled by a pair of mode indices $n, m$ for motion in the $x-y$ plane transverse to the current. In Fig. 4, we show results for the current density $j_{z}=I_{z} / W W^{\prime}$ in the $T=0$ limit, including a small thermal broadening in the numerics to improve the stability of the calculation.

We see that the numerical data are well described by the analytical result (10), with the charge renormalization factor $\kappa=0.775$ from Eq. (9). That analytical formula was derived upon linearization in $\boldsymbol{k}$ and $\boldsymbol{A}$. A more accurate calculation [46], that includes the nonlinear terms in the BDG Hamiltonian, gives $\kappa=0.750$, so the simple formula (9) is quite accurate.

Extensions.-We mention extension of our findings that may help in observing the equilibrium CME in an experiment. A first extension is to smaller flux biases in the $\mathcal{N}=2$ regime, when two pairs of charge-conjugate cones remain gapless. The supercurrent is then given by

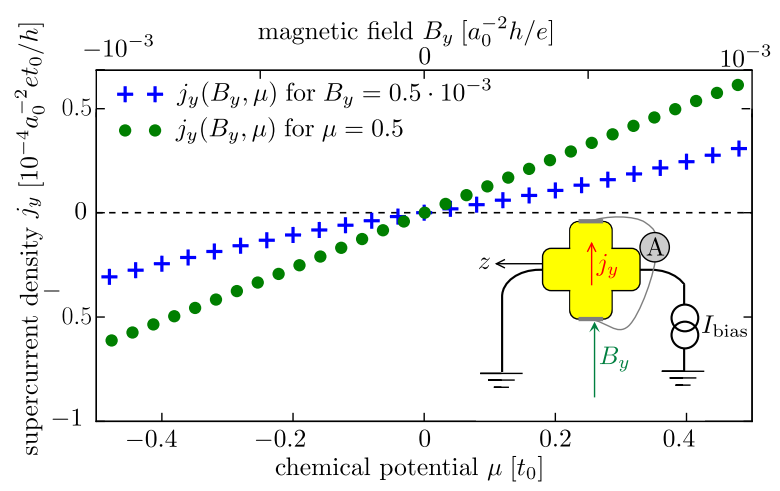

FIG. 5. Same as Fig. 4 but using the current-biased circuit shown in the inset. No antisymmetrization of the data is needed because the measured current is perpendicular to the current bias.

$\frac{\partial j_{z}}{\partial B_{z}}=\left(\kappa_{+}-\kappa_{-}\right) \frac{e^{2}}{h^{2}} \mu, \quad \kappa_{ \pm}=\sqrt{1-\Delta_{0}^{2} /(\beta \pm \Lambda)^{2}}$,

so the CME can be observed without fully gapping out one pair of cones.

A second extension is to a current-biased, rather than flux-biased circuit, with the applied magnetic field $B_{y}$ perpendicular to the current bias $j_{0}$ in the $z$ direction. The current bias then drives the Weyl superconductor into the $\mathcal{N}=1$ phase via the vector potential component $A_{z}=\mu_{0} \lambda^{2} j_{0} \equiv \Lambda / e$, with $\lambda$ the London penetration depth [56]. The analytical theory for this alternative configuration is more complicated, and not given here, but numerical results are shown in Fig. 5. While the effect is smaller than in the flux-biased configuration, it is not superimposed on a large background supercurrent so it might be more easily observed.

A third extension concerns the inclusion of disorder. Our analysis is simplified by the assumption of a clean slab, without disorder. We expect that the chirality of the zeroth Landau level will protect the equilibrium CME from degradation by impurity scattering, in much the same way as the nonequilibrium CME is protected.

Conclusion.-We have shown how the chiral anomaly of an unpaired Weyl cone can be accessed in equilibrium in a superconducting Weyl semimetal. A flux bias drives the system to a state with a single charge-conjugate pair of Weyl cones, that responds to an applied magnetic field as a single species of Weyl fermions. The cancellation of the CME for left-handed and right-handed Weyl fermions is removed, resulting in an equilibrium current along the field lines. The predicted size of the induced current is the same as that of the nonequilibrium CME, up to a charge renormalization of order unity, and since that dynamical effect has been observed [9-13], the static counterpart should be observable as well-perhaps even more easily because decoherence and relaxation play no role. 
In closing, we note that the chiral anomaly in a crystal was originally proposed [6] as a condensed matter realization of an effect from relativistic quantum mechanics and has since been an inspiration in particle physics and cosmology [57-60]. The doorway to single-cone physics that we have opened here might well play a similar role.

We have benefited from discussions with P. Baireuther, V. Cheianov, V. Ostroukh, C. Saçlıŏlu, and B. Tarasinski. This research was supported by the Netherlands Organization for Scientific Research (NWO/OCW), an ERC Synergy Grant, by funds of the Erdal İnönü chair, and by the TÜBİTAK Grant No. 114F163.

*adagideli@sabanciuniv.edu

[1] B. A. Bernevig, It's been a Weyl coming, Nat. Phys. 11, 698 (2015).

[2] D. Ciudad, Weyl fermions: Massless yet real, Nat. Mater. 14, 863 (2015).

[3] S. Jia, S.-Y. Xu, and M. Z. Hasan, Weyl semimetals, Fermi arcs and chiral anomalies, Nat. Mater. 15, 1140 (2016).

[4] A. A. Burkov, Topological semimetals, Nat. Mater. 15, 1145 (2016).

[5] S. Rao, Weyl semi-metals: A short review, J. Indian Inst. Sci. 96, 145 (2016).

[6] H. B. Nielsen and M. Ninomiya, The Adler-Bell-Jackiw anomaly and Weyl fermions in a crystal, Phys. Lett. B 130, 389 (1983).

[7] D. Kharzeev, The chiral magnetic effect and anomalyinduced transport, Prog. Part. Nucl. Phys. 75, 133 (2014).

[8] A. A. Burkov, Chiral anomaly and transport in Weyl metals, J. Phys. Condens. Matter 27, 113201 (2015).

[9] H.-J. Kim, K.-S. Kim, J.-F. Wang, M. Sasaki, N. Satoh, A. Ohnishi, M. Kitaura, M. Yang, and L. Li, Dirac versus Weyl Fermions in Topological Insulators: Adler-Bell-Jackiw Anomaly in Transport Phenomena, Phys. Rev. Lett. 111, 246603 (2013).

[10] J. Xiong, S. K. Kushwaha, T. Liang, J. W. Krizan, M. Hirschberger, W. Wang, R. J. Cava, and N. P. Ong, Evidence for the chiral anomaly in the Dirac semimetal $\mathrm{Na}_{3} \mathrm{Bi}$, Science 350, 413 (2015).

[11] X. Huang, L. Zhao, Y. Long, P. Wang, D. Chen, Z. Yang, H. Liang, M. Xue, H. Weng, Z. Fang, X. Dai, and Genfu Chen, Observation of the Chiral-Anomaly-Induced Negative Magnetoresistance in 3D Weyl Semimetal TaAs, Phys. Rev. X 5, 031023 (2015).

[12] C.-Z. Li, L.-X. Wang, H. Liu, J. Wang, Z.-M. Liao, and D.-P. Yu, Giant negative magnetoresistance induced by the chiral anomaly in individual $\mathrm{Cd}_{3} \mathrm{As}_{2}$ nanowires, Nat. Commun. 6, 10137 (2015).

[13] Q. Li, D. E. Kharzeev, C. Zhang, Y. Huang, I. Pletikosić, A. V. Fedorov, R. D. Zhong, J. A. Schneeloch, G. D. Gu, and T. Valla, Chiral magnetic effect in $\mathrm{ZrTe}_{5}$, Nat. Phys. 12, 550 (2016).

[14] M. M. Vazifeh and M. Franz, Electromagnetic Response of Weyl Semimetals, Phys. Rev. Lett. 111, 027201 (2013).
[15] J. Zhou, H. Jiang, Q. Niu, and J. Shi, Topological invariants of metals and the related physical effects, Chin. Phys. Lett. 30, 027101 (2013).

[16] Y. Chen, S. Wu, and A. A. Burkov, Axion response in Weyl semimetals, Phys. Rev. B 88, 125105 (2013).

[17] P. Goswami and S. Tewari, Axionic field theory of $(3+1)-$ dimensional Weyl semimetals, Phys. Rev. B 88, 245107 (2013).

[18] G. Basar, D. E. Kharzeev, and H.-U. Yee, Triangle anomaly in Weyl semimetals, Phys. Rev. B 89, 035142 (2014).

[19] M.-C. Chang and M.-F. Yang, Chiral magnetic effect in a two-band lattice model of Weyl semimetal, Phys. Rev. B 91, 115203 (2015).

[20] J. Ma and D. A. Pesin, Chiral magnetic effect and natural optical activity in metals with or without Weyl points, Phys. Rev. B 92, 235205 (2015).

[21] Y. Alavirad and J. D. Sau, Role of boundary conditions, topology, and disorder in the chiral magnetic effect in Weyl semimetals, Phys. Rev. B 94, 115160 (2016).

[22] S. Zhong, J. E. Moore, and I. Souza, Gyrotropic Magnetic Effect and the Magnetic Moment on the Fermi Surface, Phys. Rev. Lett. 116, 077201 (2016).

[23] P. Baireuther, J. A. Hutasoit, J Tworzydło, and C. W. J. Beenakker, Scattering theory of the chiral magnetic effect in a Weyl semimetal: interplay of bulk Weyl cones and surface Fermi arcs, New J. Phys. 18, 045009 (2016).

[24] M. A. Zubkov, Absence of equilibrium chiral magnetic effect, Phys. Rev. D 93, 105036 (2016).

[25] L. S. Levitov, Yu. V. Nazarov, and G. M. Eliashberg, Magnetostatics of superconductors without an inversion center, JETP Lett. 41, 445 (1985).

[26] Yu. V. Nazarov, Instability due to magnetically induced currents, Sov. Phys. JETP 64, 193 (1986).

[27] V. P. Mineev, Magnetostatics and optics of noncentrosymmetric metals, Phys. Rev. B 88, 134514 (2013).

[28] G. E. Volovik, The Universe in a Helium Droplet (Clarendon Press, Oxford, 2003).

[29] P. Baireuther, J. Tworzydło, M. Breitkreiz, İ Adagideli, and C. W. J. Beenakker, Weyl-Majorana solenoid, New J. Phys. 19, 025006 (2017).

[30] K.-Y. Yang, Y.-M. Lu, and Y. Ran, Quantum Hall effects in a Weyl semimetal: Possible application in pyrochlore iridates, Phys. Rev. B 84, 075129 (2011).

[31] T. Meng and L. Balents, Weyl superconductors, Phys. Rev. B 86, 054504 (2012).

[32] G. Bednik, A. A. Zyuzin, and A. A. Burkov, Superconductivity in Weyl metals, Phys. Rev. B 92, 035153 (2015).

[33] G. Y. Cho, J. H. Bardarson, Y.-M. Lu, and J. E. Moore, Superconductivity of doped Weyl semimetals: Finitemomentum pairing and electronic analog of the ${ }^{3} \mathrm{He}-\mathrm{A}$ phase, Phys. Rev. B 86, 214514 (2012).

[34] V. Shivamoggi and M. J. Gilbert, Weyl phases in pointgroup symmetric superconductors, Phys. Rev. B 88, 134504 (2013).

[35] H. Wei, S.-P. Chao, and V. Aji, Odd-parity superconductivity in Weyl semimetals, Phys. Rev. B 89, 014506 (2014).

[36] P. Hosur, X. Dai, Z. Fang, and X.-L. Qi, Time-reversalinvariant topological superconductivity in doped Weyl semimetals, Phys. Rev. B 90, 045130 (2014). 
[37] S. Kobayashi and M. Sato, Topological Superconductivity in Dirac Semimetals, Phys. Rev. Lett. 115, 187001 (2015).

[38] T. Zhou, Y. Gao, and Z. D. Wang, Superconductivity in doped inversion-symmetric Weyl semimetals, Phys. Rev. B 93, 094517 (2016).

[39] R. Wang, L. Hao, B. Wang, and C. S. Ting, Quantum anomalies in superconducting Weyl metals, Phys. Rev. B 93, 184511 (2016).

[40] T. Hashimoto, S. Kobayashi, Y. Tanaka, and M. Sato, Superconductivity in doped Dirac semimetals, Phys. Rev. B 94, 014510 (2016).

[41] M. Alidoust, K. Halterman, and A. A. Zyuzin, Superconductivity in type-II Weyl semimetals, Phys. Rev. B 95, 155124 (2017).

[42] Z. Faraei and S. A. Jafari, Superconducting proximity in three dimensional Dirac materials: odd-frequency, psueudoscalar, pseudo-vector and tensor-valued superconducting orders, arXiv:1612.06327.

[43] For the tight-binding calculations, we used the Kwant toolbox: C. W. Groth, M. Wimmer, A. R. Akhmerov, and X. Waintal, KWANT: A software package for quantum transport, New J. Phys. 16, 063065 (2014).

[44] In weak magnetic fields, for magnetic lengths $l_{m}=\sqrt{\hbar / e B}$ greater than the thickness $W$ of the slab, the applied magnetic field $B$ (in the $z$ direction, parallel to the slab) is screened by a current $j_{y}=-\left(B / \mu_{0} \lambda\right) \sinh (x / \lambda) /$ $\cosh (W / 2 \lambda)$. We neglect the screening current, first, because it is perpendicular to the CME response in the $z$ direction, and second, because its effect on the band structure (by reducing the vector potential on the surface below its unscreened value of $B W / 2$ ) is of order $1-$ $(2 \lambda / W) \tanh (W / 2 \lambda) \simeq(W / \lambda)^{2} \ll 1$ for $W$ small compared to the London penetration length $\lambda$.

[45] A stronger magnetic field, with $l_{m}<W$, may induce vortices in the order parameter, assuming a type II superconductor. We take a uniform order parameter in our analysis, however, the numerical data in Fig. 4 shows that our results extend down to the lowest fields with $l_{m} \gg W$, when vortex formation is suppressed.

[46] See Supplemental Material, Appendix B at http://link.aps .org/supplemental/10.1103/PhysRevLett.118.207701 for details of the calculation of the charge renormalization factor, including all nonlinearities in $\boldsymbol{k}$ and $\boldsymbol{A}$.

[47] See Supplemental Material, Appendix D at http://link.aps .org/supplemental/10.1103/PhysRevLett.118.207701 for a more formal derivation of the effective charge formula (10) for the equilibrium $\mathrm{CME}$.

[48] X. Wan, A. M. Turner, A. Vishwanath, and S. Y. Savrasov, Topological semimetal and Fermi-arc surface states in the electronic structure of pyrochlore iridates, Phys. Rev. B 83, 205101 (2011).

[49] The chirality $\chi$ of a Weyl cone determines the sign of the dispersion of the zeroth Landau level in a magnetic field: $\operatorname{sign}\left(d E / d k_{z}\right)=\chi \operatorname{sign}\left(B_{z}\right)$. In the flux-biased Weyl superconductor $\chi=-\operatorname{sign} \Lambda$, as one can see in Fig. 3 .

[50] F. D. M. Haldane, Attachment of surface Fermi Arcs to the bulk Fermi surface: Fermi-level plumbing in topological metals, arXiv:1401.0529.

[51] Y. Li and F. D. M. Haldane, Topological nodal Cooper pairing in doped Weyl metals, arXiv:1510.01730.

[52] See Supplemental Material, Appendix C at http://link.aps .org/supplemental/10.1103/PhysRevLett.118.207701 for a detailed calculation of the approximately neutral current carried by the surface Fermi arc.

[53] D. I. Pikulin, A. Chen, and M. Franz, Chiral Anomaly from Strain-Induced Gauge Fields in Dirac and Weyl Semimetals, Phys. Rev. X 6, 041021 (2016).

[54] A. G. Grushin, J. W. F. Venderbos, A. Vishwanath, and R. Ilan, Inhomogeneous Weyl and Dirac Semimetals: Transport in Axial Magnetic Fields and Fermi Arc Surface States from Pseudo-Landau Levels, Phys. Rev. X 6, 041046 (2016).

[55] H. Sumiyoshi and S. Fujimoto, Torsional Chiral Magnetic Effect in a Weyl Semimetal with a Topological Defect, Phys. Rev. Lett. 116, 166601 (2016).

[56] M. Tinkham, Introduction to Superconductivity (Dover Publications, New York, 2004).

[57] A. Boyarsky, J. Fröhlich, and O. Ruchayskiy, Selfconsistent Evolution of Magnetic Fields and Chiral Asymmetry in the Early Universe, Phys. Rev. Lett. 108, 031301 (2012).

[58] P. Goswami and B. Roy, Effective field theory, chiral anomaly and vortex zero modes for odd parity topological superconducting state of three dimensional Dirac materials, arXiv: 1211.4023.

[59] V. A. Miransky and I. A. Shovkovy, Quantum field theory in a magnetic field: From quantum chromodynamics to graphene and Dirac semimetals, Phys. Rep. 576, 1 (2015).

[60] M. Stone and P. Lopes, Effective action and electromagnetic response of topological superconductors and Majoranamass Weyl fermions, Phys. Rev. B 93, 174501 (2016). 